\title{
CORRELATION-INDUCTION TECHNIQUES FOR FITTING SECOND-ORDER METAMODELS IN SIMULATION EXPERIMENTS
}

\author{
Jeffrey D. Tew \\ Department of Industrial Enginecring and Operations Research \\ Virginia Polytechnic Institute and State University \\ Blacksburg, Virginia 24061-0118
}

\section{ABSTRACT}

This paper identifies two correlation-based strategies for designing a simulation experiment to estimate a second-order metamodel of the relationship between the levels of the input factors and the response of interest. Both strategies are shown to be superior to the method of independent random number streams. In the past, correlation-based strategies for metamodel estimation in simulation experiments has focused on first-order metamodels. However, in many simulation experiments it is reasonable to expect that the relationship between the levels of the input factors and the response of interest is better approximated by a second-order metamodel. Thus secondorder metamodels are, typically, of more interest to the simulation analyst. Both proposed strategies use the variance reduction technique of common random numbers to induce positive correlations between responses across design points and antithetic variates across replicates. For a large class of experimental designs and with respect to a variety of optimality criteria, both strategies are shown to give better estimates of the vector of unknown coefficients in the metamodel than the method of independent random number streams across all design points. A numerical example is given to illustrate this point and to show that in practice, the second strategy yields better metamodel estimates than the first strategy.

\section{INTRODUCTION}

The variance reduction techniques of common random numbers and antithetic variates have been used successfully in simulation experiments that are designed to estimate an hypothesized metamodel of the mean response of interest and levels of the input factors set by the simulation analyst. (Discussions of these two variance reduction techniques are given by Bratley, Fox, and Schrage (1983) and Law and Kelton (1982).) Schruben and Margolin (1978) showed that, for first-order metamodels, the method of applying common random numbers actoss all design points in the experiment yields superior estimates of the unknown parameters in the relationship to the method of conducting all simulation runs with independent, randomly selected random number streams. Furthermore, they proposed an assignment rule that, for first-order relationships that are orthogonally blockable into two orthogonal blocks, assigns a combination of common and antithetic random number streams across the design points in the experiment. This assignment rule is superior to both the use of common random number streams alone and, of course, to the use of independent, randomly selected random number streams. The requisite assumptions as well as performance evaluations for this assignment rule have been fully documented by Schruben (1979), Schruben and Margolin (1978), Tew $(1986,1989)$ and Tew and Wilson (1989 a, b).

The major contribution of the Schruben and Margolin assignment rule was to show how common random numbers and antithetic variates could be successfully 
combined in one simulation experiment. However, their results are restricted to first-order metamodels. Our computational experience has suggested that for many simulation experiments, a second-order metamodel offers a better approximation to the true underlying relationship between the mean of the response of interest and the selected levels of the input factors. In this paper we suggest two correlation-induction strategies for simulation experiments designed to estimate a second-order metamodel. In keeping with the spirit of the Schruben and Margolin assignment rule, both strategies combine the use of common random numbers and antithetic variates in one experiment. They are shown, under certain conditions, to be superior to both the method of using independent, randomly selected random number streams across all design points and the method of common random numbers across all design points.

\section{BACKGROUND AND NOTATION}

In this section we provide the statistical framework necessary to formally define a simulation experiment and its associated metamodel. We also identify the second-order metamodel used to evaluate the correlation-induction strategies presented in Section 3.

\subsection{Simulation Experiment}

Consider a simulation experiment consisting of: (a) a univariate response variable of interest, $y,(b) \mathrm{m}$ distinct design points, and (c) $r$ replications performed at each design point. A particular design point is identified by the settings of $\mathrm{d}$ input factors, or decision variables, denoted by $\phi=\left(\phi_{\mathbf{1}}\right.$, $\left.\phi_{2}, \ldots, \phi_{d}\right)^{\prime}$ that are established without error by the simulation analyst. We assume that, in general, the relationship between the response and the selected setting of $\Phi$ has the following form:

$$
y=\mu(\phi)+\varepsilon
$$

where the error term $\varepsilon$ represents the inability of $\mu$ to determine $y$.
A simulation model is usually driven by generated streams of random numbers. These streams are sequences of real numbers scaled to the interval $[0,1]$ and constructed to appear random in nature. If $\mathrm{g}$ random number streams are used to drive the simulation model and we let $\mathbf{R}_{\mathrm{ij}}$ be the set of $\mathrm{g}$ random number-streams used at the ith design point and $\mathrm{jth}$ replicate where

$$
\begin{gathered}
\mathbf{R}_{\mathrm{ij}}=\left(\mathbf{r}_{\mathrm{ij} 1}, \mathbf{r}_{\mathrm{ij} 2}, \ldots, \mathbf{r}_{\mathrm{ijg}}\right), \\
\text { for } \mathrm{i}=1,2, \ldots, \mathrm{m} \text { and } \mathrm{j}=1,2, \ldots, \mathrm{r},
\end{gathered}
$$

$\mathbf{r}_{\mathrm{ijl}}=\left(\mathbf{r}_{\mathrm{ijl} 1 \mathrm{l}}, \mathrm{r}_{\mathrm{ij} \mid 2, \ldots}, \ldots\right)^{\prime}(\mathrm{l}=1,2, \ldots, \mathrm{g})$ is the lth, potentially infinite, sequence of random numbers used to drive the simulation model at the ith design point and jth replicate, then the model above can be rewritten as

$$
\mathrm{y}_{\mathrm{ij}}\left(\mathbf{R}_{\mathrm{ij}}\right)=\mu\left(\Phi_{\mathrm{i}}\right)+\varepsilon_{\mathrm{ij}}\left(\mathbf{R}_{\mathrm{ij}}\right)
$$

$$
\text { for } i=1,2, \ldots, m \text { and } j=1,2, \ldots, r \text {, }
$$

where $y_{i j}$ is the response from the ith design point and the jth replicate, $\Phi_{i}$ is the setting of the $d$ factors at the ith design point, and $\varepsilon_{\mathrm{ij}}$ is the error at the ith design point and $\mathrm{jth}$ replicate. Typically, $\mu$ is unknown and needs to be estimated. The estimation process usually involves two steps: (a) hypothesize a functional approximation of $\mu$ and (b) estimate any unknown parameters in the hypothesized approximation.

For example, if we assume that $\mu$ is first-order and linear in the unknown parameters then the equation above can be written as

$$
\begin{gathered}
y_{i j}\left(\mathbf{R}_{i j}\right)=\beta_{o}+\sum_{k=1}^{p-1} \beta_{k} x_{k}\left(\phi_{i}\right)+\varepsilon_{i j}\left(R_{i j}\right) \\
\text { for } i=1,2, \ldots, m \text { and } j=1,2, \ldots, r
\end{gathered}
$$

where $\beta=\left(\beta_{o}, \beta_{1}, \ldots, \beta_{p-1}\right)^{\prime}$ is the $(p \times 1)$ vector of unknown metamodel coefficients; $x_{k}(k=1,2, \ldots, p-1)$ represent known functions of these settings; and $y_{i j}, \Phi_{i}$, and $\varepsilon_{i j}$ are defined above. 
Equation (2) can be written in short-hand matrix notation as:

$$
\mathbf{y}_{\mathbf{j}}\left(\mathbf{R}_{\mathrm{j}}\right)=\mathbf{X} \beta+\varepsilon_{\mathrm{j}}\left(\mathbf{R}_{\mathrm{j}}\right), \text { for } \mathrm{j}=1,2, \ldots, \mathbf{r},
$$

where $\mathbf{y}_{\mathrm{j}}=\left(\mathrm{y}_{1 \mathrm{j}}, \mathrm{y}_{2 \mathrm{j}}, \ldots, \mathrm{y}_{\mathrm{mj}}\right)^{\prime}, \mathbf{X}$ is the $(\mathrm{m} \times \mathrm{p})$ design matrix whose first column is all ones and whose $(i, j+1)$ th element is $x_{j}\left(\phi_{j}\right)(i=1,2, \ldots, m$ and $j=1,2, \ldots, p-1), \beta$ is defined above, $\underset{\mathrm{f}}{\varepsilon_{j}}=\left(\varepsilon_{1 j}, \varepsilon_{2 j}, \ldots, \varepsilon_{\mathrm{mj}}\right)^{\prime}$, and $\mathbf{R}_{\mathrm{j}}=\left(\mathbf{R}_{1 \mathrm{j}}, \mathbf{R}_{2 \mathrm{j}}, \ldots, \mathbf{R}_{\mathrm{mj}}\right)^{\prime}$.

We also assume that $\underset{\varepsilon_{j}}{\varepsilon_{j}}(j=1,2, \ldots, r)$ has the following multivariate normal distribution:

$$
\varepsilon_{\mathrm{j}} \sim \mathrm{N}_{\mathrm{m}}(\mathbf{0}, \Sigma), \quad \text { for } \mathrm{j}=1,2, \ldots, \mathrm{r},
$$

where 0 is a $(\mathrm{m} \times 1)$ vector of zeros and $\Sigma$ is a $(\mathrm{m} \times \mathrm{m})$ covariance matrix.

From (4) we get that

$$
\mathbf{y}_{\mathrm{j}} \sim \mathrm{N}_{\mathrm{m}}(\mathbf{X} \beta, \Sigma), \quad \text { for } \mathrm{j}=1,2, \ldots, \mathrm{r}
$$

Under these assumptions, and for $m>p$, the least-squares estimate of $\beta$; which is given by

$$
\beta=\left(X^{X}\right)^{-1} X^{\prime} \bar{y}
$$
Where $\overline{\mathbf{y}}=\sum_{\mathrm{j}=1}^{\mathrm{r}} \mathbf{y}_{\mathrm{j}}$, has the following multivariate normal
distribution:

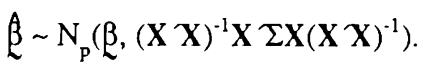

\subsection{Second-Order Metamodel}

In this section we identify the second-order metamodel that will be used for purposes of illustration and evaluation for the four correlation-induction strategies presented in the next section.

Consider the following second-order, two factor metamodel:

$$
\mathrm{y}_{\mathrm{ij}}=\gamma_{\mathrm{o}}+\gamma_{1} \phi_{1 \mathrm{i}}+\gamma_{2} \phi_{2 \mathrm{i}}+\gamma_{3} \phi_{1 \mathrm{i}}^{2}+\gamma_{4} \phi_{2 \mathrm{i}}^{2}+\gamma_{5} \phi_{1 \mathrm{i}} \phi_{2 \mathrm{i}}+\varepsilon_{\mathrm{ij}}
$$

$$
\text { for } \mathrm{i}=1,2, \ldots, \mathrm{m} \text { and } \mathrm{j}=1,2, \ldots, \mathrm{r} \text {, }
$$

where $\phi_{1 \mathrm{i}}$ and $\phi_{2 \mathrm{i}}$ are the levels of the two factors at the ith design point, $\gamma_{i}(i=0,1, \ldots, 5)$ are the unknown model coefficients, and $y_{i j}$ and $\varepsilon_{i j}$ are defined in Section 2.1. Since (8) is a second order metamodel we should select 3 levels for each factor in order to obtain estimates of all parameters in the metamodel.

If we select the levels of $\phi_{1}$ and $\phi_{2}$ so that they are evenly spaced then we can simplify the estimation of $\underset{\gamma}{\gamma}=\left(\gamma_{0}\right.$, $\left.\gamma_{1}, \ldots, \gamma_{5}\right)^{\prime}$ by a suitable coding of the factors $\phi_{1}$ and $\phi_{2}$. For example, if we code $\phi_{1}$ to $x_{1}$ and $\phi_{2}$ to $x_{2}$, where the $x_{i}$ 's ( $i=$ $1,2)$ take on a 0 value in the center of the design and -1 and +1 at the low and high level, respectively, the levels of the $\phi$ i's ( $i=1,2)$ being spaced evenly, the design matrix for the complete $3^{2}$ factorial experiment is given by

$$
\mathbf{X}=\left[\begin{array}{cccccc}
1 & \mathrm{x}_{1 \mathrm{i}} & \mathrm{x}_{2 \mathrm{i}} & \mathrm{x}_{1 \mathrm{i}}^{2} & \mathrm{x}_{2 \mathrm{i}}^{2} & \mathrm{x}_{1 \mathrm{i}} \mathrm{x}_{2 \mathrm{i}} \\
1 & -1 & -1 & 1 & 1 & 1 \\
1 & -1 & 0 & 1 & 0 & 0 \\
1 & 0 & -1 & 0 & 1 & 0 \\
1 & 0 & 0 & 0 & 0 & 0 \\
1 & 0 & 1 & 0 & 1 & 0 \\
1 & 1 & -1 & 1 & 1 & -1 \\
1 & 1 & 0 & 1 & 0 & 0 \\
1 & 1 & 1 & 1 & 1 & 1
\end{array}\right]
$$

The matrix (XX) is not diagonal when the metamodel is written in this form. However, if we rewrite the metamodel as

$$
\begin{aligned}
& y_{i j}=\beta_{o}+\beta_{1} x_{1 i}+\beta_{2} x_{2 i}+\beta_{3}\left(x_{1 i}^{2}-\bar{x}_{1}^{2}\right)+ \\
& +\beta_{4}\left(x_{2 i}^{2}-\bar{x}_{2}^{2}\right)+\beta_{5} x_{1 i} x_{2 i}+\varepsilon_{i j}, \\
& \quad \text { for } i=1,2, \ldots, m \text { and } j=1,2, \ldots, r,
\end{aligned}
$$

where $\bar{x}_{1}^{2}-\bar{x}_{1}^{2}$ are the average values of $x_{1 \mathrm{i}}^{2}$ and $x_{2 \mathrm{i}}^{2}$, respectively. Thus, equation (9) becomes 


$\mathbf{X}=\left[\begin{array}{cccccc}1 & \mathrm{x}_{1 \mathrm{i}} & \mathrm{x}_{2 \mathrm{i}} & \left(\mathrm{x}_{1 \mathrm{i}}^{2}-\overline{\mathrm{x}}_{1}^{2}\right) & \left(\mathrm{x}_{2 \mathrm{i}}^{2}-\overline{\mathrm{x}}_{2}^{2}\right) & \mathrm{x}_{1 \mathrm{i}} \mathrm{x}_{2 \mathrm{i}} \\ 1 & -1 & -1 & 1 / 3 & 1 / 3 & 1 \\ 1 & -1 & 0 & 1 / 3 & -2 / 3 & 0 \\ 1 & 0 & -1 & -2 / 3 & 1 / 3 & 0 \\ 1 & 0 & 0 & -2 / 3 & -2 / 3 & 0 \\ 1 & 0 & 1 & -2 / 3 & 1 / 3 & 0 \\ 1 & 1 & -1 & 1 / 3 & 1 / 3 & -1 \\ 1 & 1 & 0 & 1 / 3 & -2 / 3 & 0 \\ 1 & 1 & 1 & 1 / 3 & 1 / 3 & 1\end{array}\right]$

and

$$
\mathbf{X} \mathbf{X}=\left[\begin{array}{llllll}
9 & 0 & 0 & 0 & 0 & 0 \\
0 & 6 & 0 & 0 & 0 & 0 \\
0 & 0 & 6 & 0 & 0 & 0 \\
0 & 0 & 0 & 2 & 0 & 0 \\
0 & 0 & 0 & 0 & 2 & 0 \\
0 & 0 & 0 & 0 & 0 & 4
\end{array}\right]
$$

This method for diagonalizing the $\mathbf{X}^{\prime} \mathbf{X}$ matrix is called the method of orthogonal polynomials. (See Chapter 3 of Myers (1976) for a more complete treatment of orthogonal polynomials for second order designs.)

The second order metamodel presented in equation (1) is used in the next section to compare the general efficacy of the four correlation-induction strategies considered.

\section{CORRELATION-INDUCTION STRATEGIES}

In this section we consider the following four correlation-induction strategies for estimating $\beta=\left(\beta_{o}, \beta_{1}, \ldots\right.$, $\left.\beta_{5}\right)^{\prime}$ in equation (10): (a) independent streams, (b) common streams, (c) correlated replicates I, and (d) correlated replicates II. In comparing these four strategies we show that, under certain assumptions, the last two are superior to strategies (a) and (b). Throughout the remainder of this paper we assume that there are $2 r$ replicates made at each design point.

\subsection{Independent Streams}

If we randomly select $\mathbf{R}_{\mathrm{ij}}(\mathrm{i}=1,2, \ldots, \mathrm{m}$ and $\mathrm{j}=1$, $2, \ldots, 2 r)$ and we assume that $(i=1,2, \ldots, m$ and $j=1,2, \ldots$, $2 r)$ :

$\left.\begin{array}{l}\operatorname{Var}\left(y_{i j}\left(\mathbf{R}_{i j}\right)\right)=\sigma^{2} \\ \operatorname{Cov}\left(y_{i j}\left(R_{i j}\right), y_{k l}\left(R_{k l}\right)\right)=0 \text { for } i \neq k, j \neq l ;\end{array}\right\}$

then $\Sigma_{1}=\operatorname{Cov}(\mathbf{y})=\frac{\sigma^{2}}{2 r} I_{m}$, where $I_{m}$ is the $(m \times m)$ identity matrix. Substituting this value of $\Sigma$, into (7) yields

$\operatorname{Cov}(\beta)=\frac{\sigma^{2}}{2 \mathrm{r}}\left[\begin{array}{ccccccc}1 / 9 & 0 & 0 & 0 & 0 & 0 & 0 \\ 0 & 1 / 6 & 0 & 0 & 0 & 0 & 0 \\ 0 & 0 & 1 / 6 & 0 & 0 & 0 & 0 \\ 0 & 0 & 0 & 1 / 2 & 0 & 0 & 0 \\ 0 & 0 & 0 & 0 & 1 / 2 & 0 & 0 \\ 0 & 0 & 0 & 0 & 0 & 1 / 2 & 0 \\ 0 & 0 & 0 & 0 & 0 & 0 & 1 / 4\end{array}\right]$.

\subsection{Common Streams}

If we separate $\mathbf{R}_{\mathrm{ij}}(\mathrm{i}=1,2, \ldots, \mathrm{m}$ and $\mathrm{j}=1,2, \ldots, 2 \mathrm{r}$ ) into two mutually exclusive sets of random number streams, $\left(\mathbf{R}_{1 \mathrm{ij}}, \mathbf{R}_{2 \mathrm{ij}}\right)$, such that $\mathbf{R}_{2 \mathrm{ij}}(\mathrm{i}=1,2, \ldots, \mathrm{m}$ and $\mathrm{j}=1,2, \ldots, 2 \mathrm{r})$ is independently, randomly selected across all design points and replicates, then we are assured of the presence of $\varepsilon_{\mathrm{ij}}(\mathrm{i}=$ $1,2, \ldots, m$ and $j=1,2, \ldots, 2 r$ ) in (10). (See Mihram (1974), and Tew (1989).) Hence, in inducing correlations across design points and across replicates, we use only $\mathbf{R}_{1 \mathrm{ij}}(i=1,2$, $\ldots, m$ and $j=1,2, \ldots, 2 r$ ). This technique is used for the present strategy as well as the strategies in Sections 3.3 and 3.4 .

In order to induce positive correlations across design points and retain uncorrelated replicates we use the random number assignment procedure indicated in Table 1. Further, we assume that $(i=1,2, \ldots, m ; k=1,2, \ldots, m ; j=1$, $2, \ldots, 2 \mathrm{r})$; and $\mathrm{l}=1,2, \ldots, 2 \mathrm{r}$ ): 


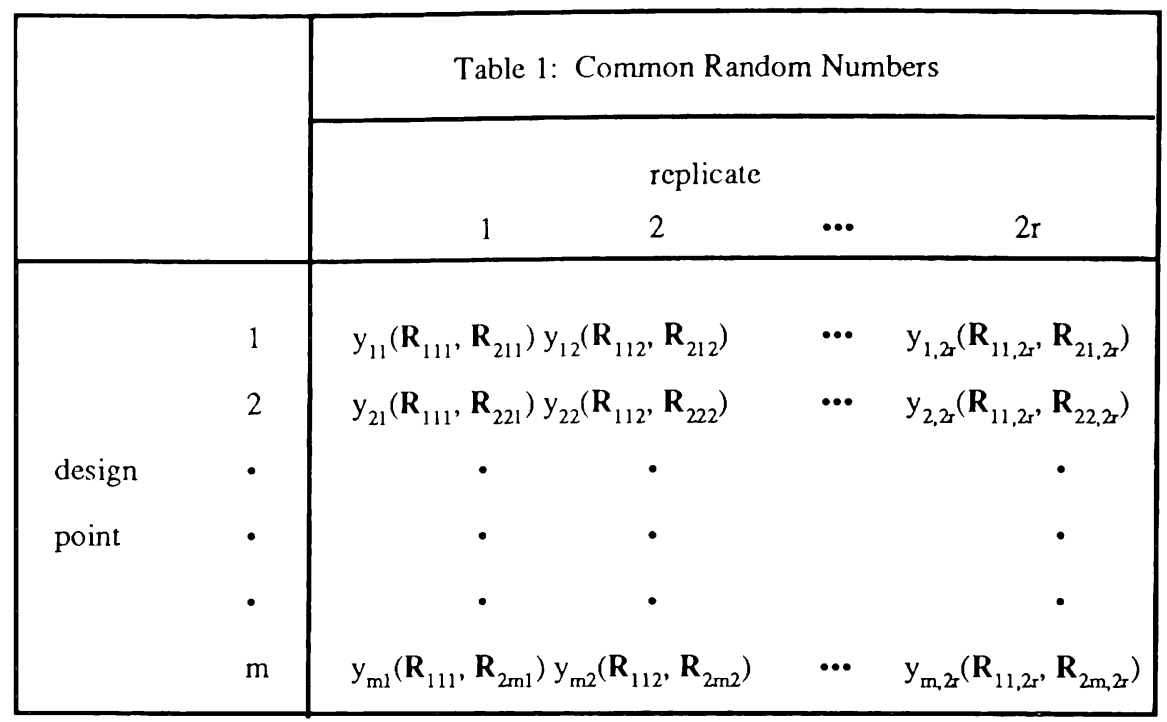

\subsection{Correlated Replicates I}

Next, consider the random number assignment

$\operatorname{Var}\left(\mathrm{y}_{\mathrm{ij}}\left(\mathbf{R}_{\mathrm{ij}}\right)\right)=\sigma^{2} ;$

$\operatorname{Cov}\left(y_{i j}\left(\mathbf{R}_{\mathrm{ij}}\right), y_{\mathrm{kj}}\left(\mathbf{R}_{\mathrm{kj}}\right)\right)=\sigma^{2} \rho_{+}$ where $0 \leq \rho_{+} \leq 1$ and $i \neq k$;

$\operatorname{Cov}\left(y_{i j}\left(\mathbf{R}_{\mathrm{ij}}\right), y_{\mathrm{kl}}\left(\mathbf{R}_{\mathrm{kl}}\right)\right)=0$

for $\mathrm{j} \neq 1$.

This assignment procedure and the assumptions of (15) yield:

$\Sigma_{2}=\operatorname{Cov}(\overline{\mathbf{y}})=\frac{\sigma^{2}\left(1-\rho_{+}\right)}{2 \mathrm{r}} \mathbf{I}_{\mathrm{m}}+\frac{\sigma^{2} \rho_{+}}{2 \mathrm{r}} \mathbf{E}_{\mathrm{m}}$,

where $E_{m}$ is the $(m \times m)$ matrix of ones. Substitution of $\Sigma_{2}$ into (7) yields

$\operatorname{Cov}(\beta)=$

$$
\frac{\sigma}{2 \tau}\left[\begin{array}{cccccc}
\frac{\left(9\left(\rho_{+}+\rho_{-}\right) / 2+\left(1-\rho_{+}\right)\right)}{9} & 0 & 0 & 0 & 0 & 0 \\
0 & \frac{\left(1-\rho_{+}\right)}{6} & 0 & 0 & 0 & 0 \\
0 & 0 & \frac{\left(1-\rho_{+}\right)}{6} & 0 & 0 & 0 \\
0 & 0 & 0 & \frac{\left(1-\rho_{+}\right)}{2} & 0 & 0 \\
0 & 0 & 0 & 0 & \frac{\left(1-\rho_{+}\right)}{2} & 0 \\
0 & 0 & 0 & 0 & 0 & \frac{\left(1-\rho_{+}\right)}{4}
\end{array}\right] .
$$

procedure indicated by Table 2 . We assume that $(i=1,2, \ldots$, $\mathrm{m} ; \mathrm{k}=1,2, \ldots, \mathrm{m} ; \mathrm{j}=1,2, \ldots, 2 \mathrm{r} ;$ and $\mathrm{l}=1,2, \ldots, 2 \mathrm{r}$ ):

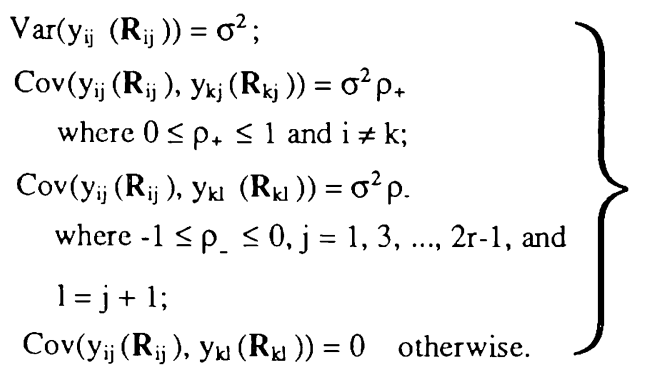

This assignment procedure, coupled with the assumptions of (18) yields

$$
\Sigma_{3}=\operatorname{Cov}(\mathbf{y})=\frac{\sigma^{2}\left(1-\rho_{+}\right)}{2 r} \mathbf{I}_{m}+\frac{\sigma^{2}\left(\rho_{1}+\rho_{-}\right)}{2} \mathbf{E}_{\mathrm{m}} .
$$




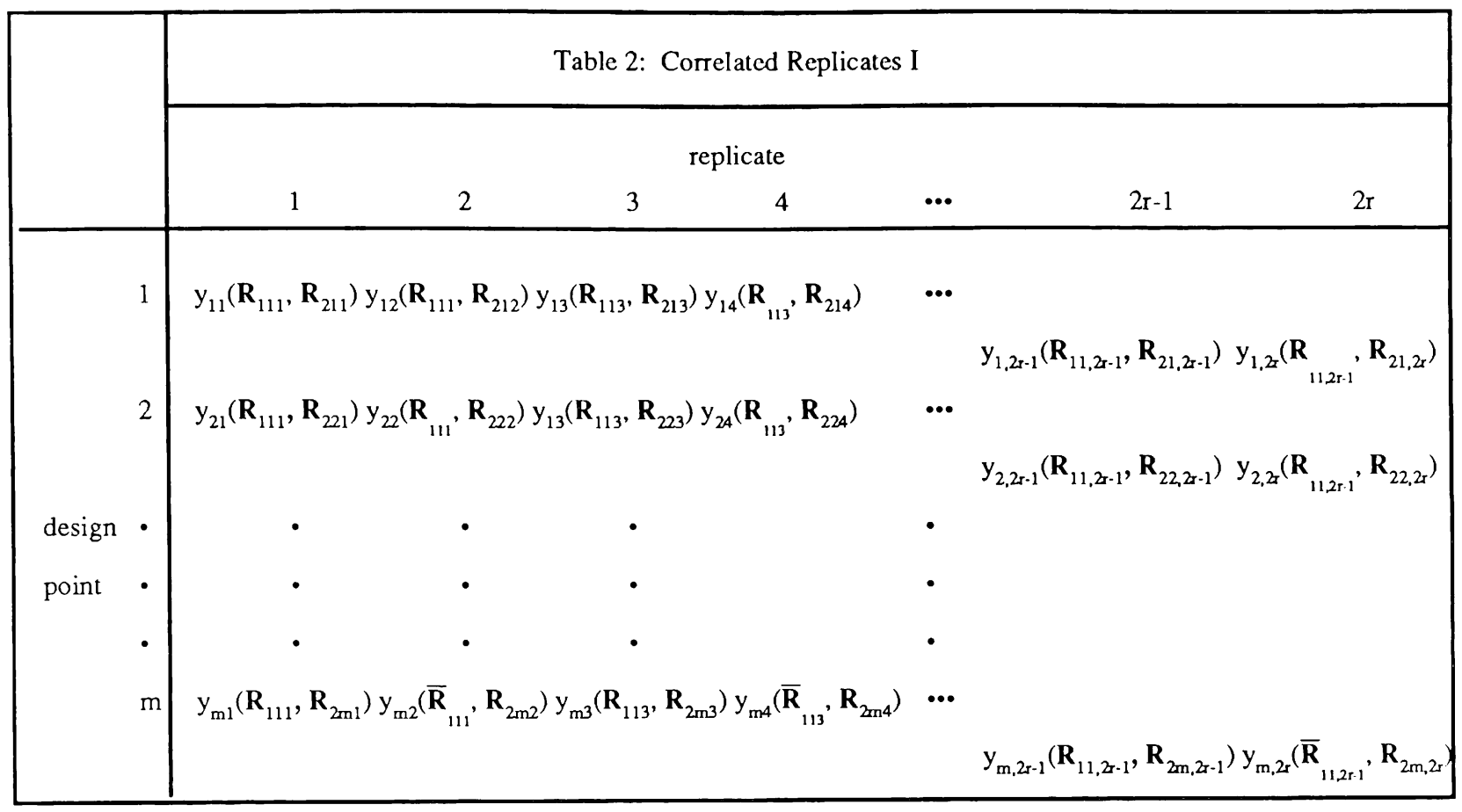

Substitution of (19) into (7) yields:

$\operatorname{Cov}(\beta)=$

$\frac{\sigma^{2}}{2 \mathrm{r}}\left[\begin{array}{cccccc}\frac{\left(9 \gamma\left(\rho_{+}+\rho_{-}\right)+\left(1-\rho_{+}\right)\right)}{9} & 0 & 0 & 0 & 0 & 0 \\ 0 & \frac{\left(1-\rho_{+}\right)}{6} & 0 & 0 & 0 & 0 \\ 0 & 0 & \frac{\left(1-\rho_{+}\right)}{6} & 0 & 0 & 0 \\ 0 & 0 & 0 & \frac{\left(1-\rho_{+}\right)}{2} & 0 & 0 \\ 0 & 0 & 0 & 0 & \frac{\left(1-\rho_{+}\right)}{2} & 0 \\ 0 & 0 & 0 & 0 & 0 & \frac{\left(1-\rho_{+}\right)}{4}\end{array}\right]$

$$
\begin{gathered}
\operatorname{Var}\left(y_{\mathrm{ij}}\left(\mathbf{R}_{\mathrm{ij}}\right)\right)=\sigma^{2} ; \\
\operatorname{Cov}\left(\mathrm{y}_{\mathrm{ij}}\left(\mathbf{R}_{\mathrm{ij}}\right), \mathrm{y}_{\mathrm{kj}}\left(\mathbf{R}_{\mathrm{kj}}\right)\right)=\sigma^{2} \rho_{+} \\
\operatorname{where} 0 \leq \rho_{+} \leq 1 \text { and } \mathrm{i} \neq \mathrm{k} ; \\
\operatorname{Cov}\left(\mathrm{y}_{\mathrm{ij}}\left(\mathbf{R}_{\mathrm{ij}}\right), \mathrm{y}_{\mathrm{kl}}\left(\mathbf{R}_{\mathrm{kl}}\right)\right)=\sigma^{2} \rho_{+} \\
\text {for } \| \mathrm{l}-\mathrm{jl}=2,4, \ldots, 2 \mathrm{r}-2 \\
\operatorname{Cov}\left(\mathrm{y}_{\mathrm{ij}}\left(\mathbf{R}_{\mathrm{ij}}\right), \mathrm{y}_{\mathrm{kl}}\left(\mathbf{R}_{\mathrm{kd}}\right)\right)=\sigma^{2} \rho \\
\text { where }-1 \leq \rho . \leq 0 \text {, and } \\
\| 1-\mathrm{jl}=1,3, \ldots, 2 \mathrm{r}-1 .
\end{gathered}
$$

This assignment procedure and the assumptions of (21), together, yield:

$$
\Sigma_{4}=\operatorname{Cov}(\overline{\mathbf{y}})=\frac{\sigma^{2}\left(1-\rho_{+}\right)}{2 \mathrm{r}} \mathbf{I}_{\mathrm{m}}+\frac{\sigma^{2}\left(\rho_{+}+\rho_{-}\right)}{2} \mathbf{E}_{\mathrm{m}} .
$$

procedure indicated by Table 3, where $\overline{\mathbf{R}}_{\mathrm{ijk}}$ is the set of random number streams antithetic to those in $\mathbf{R}_{\mathrm{ijk}}(\mathrm{i}=1,2 ; \mathrm{j}$ $=1,2, \ldots, \mathrm{m} ;$ and $\mathrm{k}=1,2, \ldots, 2 \mathrm{r}$ ).

We assume that $(i=1,2, \ldots, m ; k=1,2, \ldots, m ; j=$ $1,2, \ldots, 2 r ;$ and $l=1,2, \ldots, 2 r)$ : 


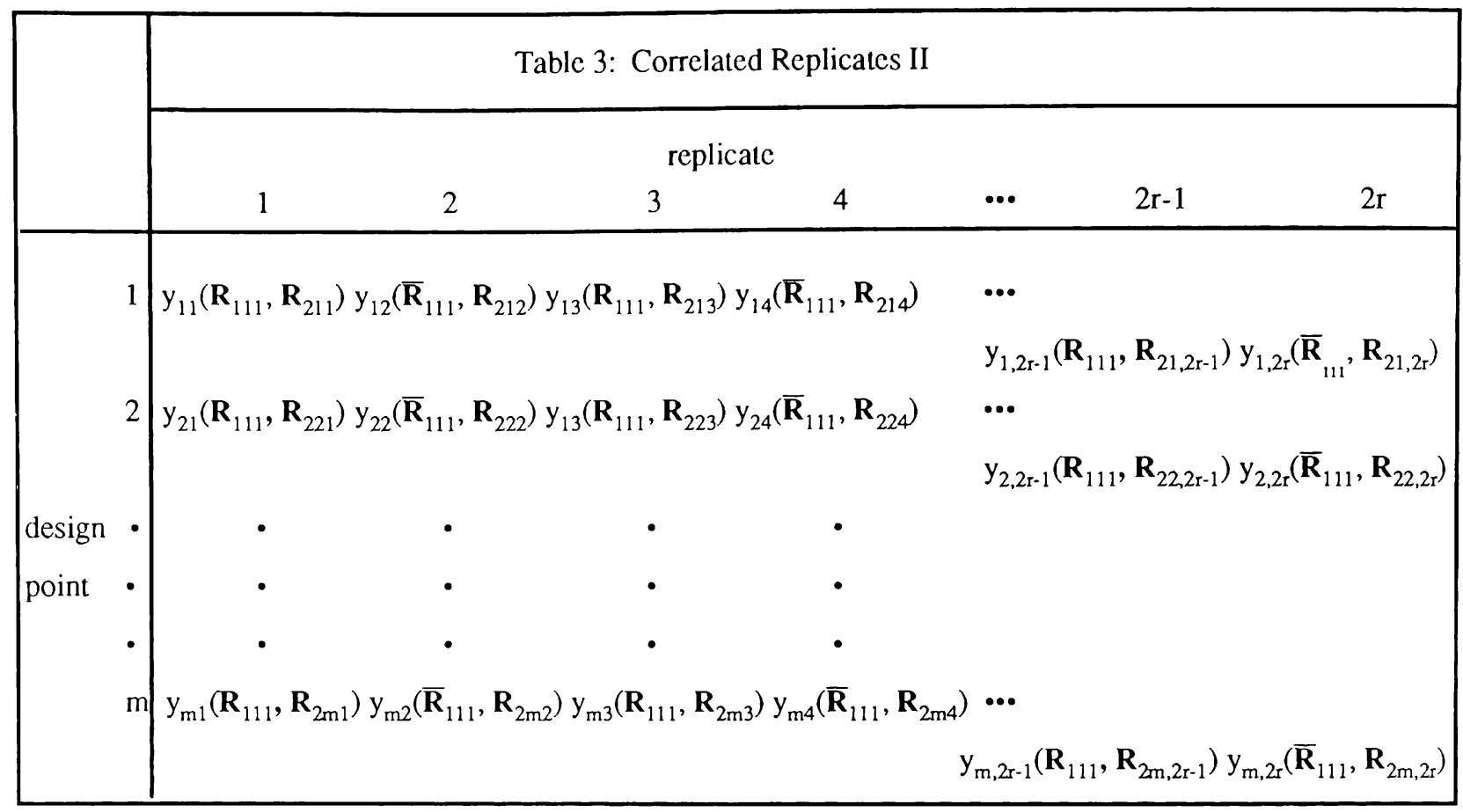

Substitution of (22) into (7) yields:

$\operatorname{Cov}(\beta)=$

$\frac{\sigma^{2}}{2 r}\left[\begin{array}{cccccc}\frac{\left(9\left(\rho_{+}+\rho_{-}\right)+\left(1-\rho_{-}\right)\right)}{9} & 0 & 0 & 0 & 0 & 0 \\ 0 & \frac{\left(1-\rho_{+}\right)}{6} & 0 & 0 & 0 & 0 \\ 0 & 0 & \frac{\left(1-\rho_{+}\right)}{6} & 0 & 0 & 0 \\ 0 & 0 & 0 & \frac{\left(1-\rho_{+}\right)}{2} & 0 & 0 \\ 0 & 0 & 0 & 0 & \frac{\left(1-\rho_{+}\right)}{2} & 0 \\ 0 & 0 & 0 & 0 & 0 & \frac{\left(1-\rho_{+}\right)}{4}\end{array}\right]$

\subsection{Comparison}

Inspection of equations (14), (17), (20), and (23) indicates that in terms of $\operatorname{Var}\left(\hat{\beta}_{i}\right)(i=0,1, \ldots, 5)$, common streams, correlated replicates I, and correlated replicates II arc: (a) all equivalent in the estimation of $\beta=\left(\beta_{1}, \beta_{2}, \ldots, \beta_{5}\right)^{\prime}$ and (b) all superior to the independent streams strategy in the estimation of $\beta_{1}=\left(\beta_{1}, \beta_{2}, \ldots, \beta_{5}\right)$. We also see that under the assumptions of (18) that $\left|\rho_{!}\right| \geq\left|\rho_{+}\right|$and $\rho_{-}<0$ are sufficient conditions for correlated replicates $I$ to be superior to common streams and independent streams. Furthermore, we see that, under the assumptions of (21), correlated replicates II is superior in the estimation of $\beta$ to common streams and independent streams if $\rho_{.}<0$. In practice we expect the condition $|\rho| \geq\left|\rho_{+}\right|$not to hold in general (see Schruben and Margolin (1978) and Tew (1986)). Thus, we expect correlated replicates II to give the best performance in the estimation of $\beta$ for the second order metamodel given by equation (10).

\section{EXAMPLE}

In this section we illustrate the implementation of the four strategies presented in Section 3. We also compare the estimators of the metamodel coefficients under these four strategies.

\subsection{The Job Shop System}

Consider the job shop example used by Tew and Wilson (1987) and similar to the one given by Nozari, Arnold, and Pegden (1987), and depicted in Figure 1. Past computations have suggested that a first-order metamodel is 
inappropriate for this model. Jobs arrive at this shop according to a Poisson process with an arrival rate of 10 per hour. All jobs enter the system through station 1. Upon completing service at station $1,80 \%$ of the jobs go to station $2,5 \%$ go to station 3 , and $15 \%$ leave the system. A job at station 2 , or station 3 , leaves the system upon completion of service. The shop admits jobs from 8:00 A.M. to 4:00 P.M. every day. However, service at each station continues until all jobs admitted on one day leave the system. Service time at station 1 is a constant and service times at stations 2 and 3 are uniformly distributed over specified ranges.

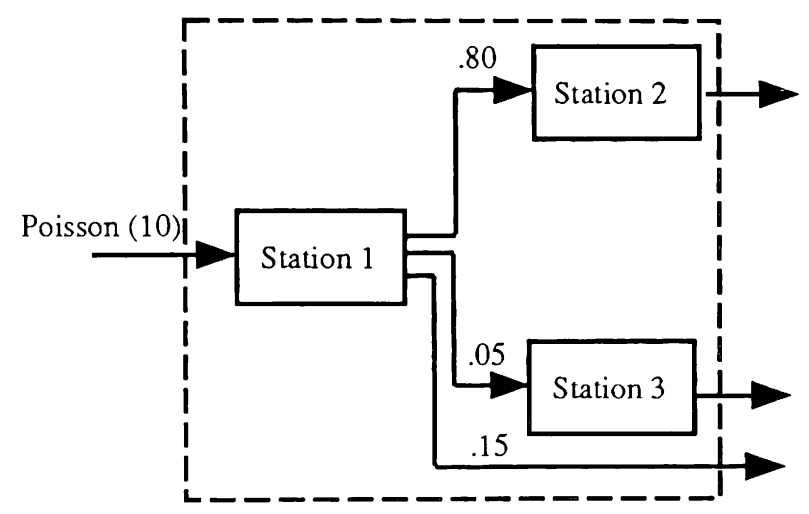

Figure 1. Job Shop System

The purpose of this example is to estimate the effects that different service time distributions have on some function of the expected system sojoum time for a job. Thus, the performance measure of interest is the daily average system sojourn time for all jobs entering the system. This estimation is done under the four strategies presented.

\subsection{The Model of the Response}

To study this system we employ the $3^{2}$ factorial design of Section 2 with the following independent variables (factors): service time distribution at station $1\left(x_{1}\right)$ and service time distribution at station $2\left(\mathrm{x}_{2}\right)$. We consider the second-order metamodel given by (10).

In simulating this system we dedicated a separate random number stream to each of the following four random components in the model: interarrival times at station $1\left(\mathbf{r}_{1}\right)$, probabilistic branching upon completion of service at station $1\left(r_{2}\right)$, service times at station $2\left(r_{3}\right)$, and service times at station $3\left(\mathbf{r}_{4}\right)$. Under common streams, correlated replicates I, and correlated replicates II, we randomly selected $\mathbf{r}_{4}$ across all design points and replicates in order to ensure the presence of $\varepsilon_{\mathrm{ij}}$ in (10).

Next, we consider the estimation of $\beta$ under each of the four strategies for conducting simulation experiments mentioned above. In each case, 18 estimates of $\beta$ are obtained by replicating the basic 9-point experiment 18 times. The sample covariance matrix based on these 18 estimates is used as an external estimate of the covariance matrix of the estimator of $\beta$.

\subsection{Numerical Results}

For the independent streams strategy we get

$\operatorname{Cov} \underset{\sim}{\hat{\beta}})=\left[\begin{array}{rrrrrr}58.55 & -6.85 & 2.79 & 0.76 & -53.09 & -25.96 \\ -6.85 & 36.05 & 11.05 & 38.74 & -29.40 & 24.03 \\ 2.79 & 11.05 & 64.41 & 26.86 & 19.08 & 18.86 \\ 0.76 & 38.74 & 26.86 & 266.26 & -26.95 & -6.09 \\ -53.09 & -29.40 & 29.08 & -26.95 & 236.31 & -14.90 \\ -25.96 & 24.03 & 18.86 & -6.09 & -14.90 & 77.45\end{array}\right]$

For the common streams strategy we get

$\operatorname{Cov}(\underset{\sim}{\hat{\beta}})=\left[\begin{array}{rrrrrr}569.85 & 156.29 & 113.00 & -237.85 & -46.25 & -150.28 \\ 156.29 & 79.35 & 21.62 & -64.74 & 7.79 & -31.75 \\ 113.00 & 21.62 & 30.45 & -41.87 & -5.81 & -35.54 \\ -237.85 & -64.74 & -41.87 & 124.08 & 22.55 & 56.78 \\ -46.25 & 7.79 & -5.81 & 22.55 & 31.79 & 13.64 \\ -150.28 & -31.75 & -35.54 & 56.78 & 13.64 & 45.45\end{array}\right]$

For the correlated replicates I strategy we get

$\operatorname{Cov}(\underset{\sim}{\hat{\beta}})=\left[\begin{array}{rrrrrr}257.40 & 37.58 & 44.61 & -115.56 & -61.14 & -67.35 \\ 37.58 & 51.29 & 3.19 & 2.47 & 18.25 & -4.25 \\ 44.61 & 3.19 & 17.52 & -0.88 & -7.94 & -19.18 \\ -115.56 & 2.47 & -0.88 & 111.80 & 47.97 & 16.01 \\ -61.14 & 18.25 & -7.94 & 47.97 & 36.21 & 14.73 \\ -67.35 & -4.25 & -19.18 & 16.01 & 14.73 & 25.11\end{array}\right]$

Finally, for the correlated replicates II strategy we get

$\operatorname{Côv}(\underset{\sim}{\beta})=\left[\begin{array}{rrrrrr}359.27 & 33.36 & 20.10 & -279.81 & -146.53 & -65.17 \\ 33.36 & 3.12 & 1.86 & -26.00 & -13.61 & -6.03 \\ 20.10 & 1.86 & 1.15 & -15.65 & -8.17 & -3.66 \\ -279.81 & -26.00 & -15.65 & 218.02 & 114.15 & 50.74 \\ -146.53 & -13.61 & -8.17 & 114.15 & 59.87 & 26.56 \\ -65.17 & -6.03 & -3.66 & 50.74 & 26.56 & 11.86\end{array}\right]$ 


\section{CONCLUSIONS}

Although any statistical comparison of the sample covariance matrices in Section 4.3 will have low power due to the relatively small number of replications the results strongly suggest that the correlated replicates strategies yield superior performance in the estimation of $\beta$ for the metamodel given by (10). However, caution should be taken in that the last three sample covariance matrices in Section 4.3 may be biased because the replicates are correlated. Nevertheless, the results are promising and warrant further, more extensive investigation.

\section{REFERENCES}

Bratley, P., Fox, B. L. and Schrage, L. (1983). A Guide to Simulation. Springer-Verlag, New York.

Law, A. M. and Kelton, W. D. (1982). Simulation Modeling and Analysis. McGraw-Hill, New York.

Mihram, G. A. (1974). Blocking in simular experimental designs. Journal of Statistical Computation and Simulation, 3, 29-32.

Myers, R. H. (1976). Response Surface Methodology. Allyn and Bacon, Boston, Massachusetts.

Schruben, L. W. (1979). Designing correlation induction strategies for simulation experiments. In: Current Issues in Computer Simulation (N. R. Adam and A. Dogramaci eds.). Academic Press, New York, 235-256.

Schruben, L. W. and Margolin, B. H. (1978). Pscudorandom number assignment in statistically designed simulation and distribution sampling experiments. Journal of the American Statistical Association, 73, 504-525.

Tew, J. D. (1986). Metamodel estimation under correlation methods for simulation experiments. Unpublished Ph.D. dissertation, School of Industrial Engincering, Purdue University, West Lafayette, Indiana.
Tew, J. D. (1989). Correlated replicates designs for first-order metamodel estimation in simulation experiments. Technical Report VTR 8903. Department of Industrial Engineering and Operations Research, Virginia Polytechnic Institute and State University, Blacksburg, Virginia.

Tew, J. D. and Wilson, J. R. (1989a). Validation of statistical analysis methods for the Schruben-Margolin correlation-induction strategy. Technical Report VTR 8701 (revised). Department of Industrial Engineering and Operations Research, Virginia Polytechnic Institute and State University, Blacksburg, Virginia.

Tew, J. D. and Wilson, J. R. (1989b). Estimating simulation metamodels using integrated variance reduction techniques. Technical Report VTR 8902. Department of Industrial Engineering and Operations Research, Virginia Polytechnic Institute and State University, Blacksburg, Virginia.

\section{AUTHORS' BIOGRAPHIES}

JEFFREY D. TEW is an Assistant Professor in the Department of Industrial Engineering and Operations Research at Virginia Polytechnic Institute and State University. He received a B. S. in mathematics from Purduc University in 1979, a M.S. in statistics from Purdue University in 1981, and a Ph.D. in industrial engineering from Purdue University in 1986. His current rescarch interests include simulation optimization and design of experiments. He is a member of ASA, IIE, IMS, ORSA, and SCS.

Jeffrey D. Tew

Department of IEOR

Virginia Polytechnic Institute and State University

Blacksburg, Virginia 24061-0118

(703) 231-7099 